5 Research Square

\title{
COVID-19: Does lifestyle intervention improve the course of the disease? A case series and literature review
}

Salete da Silva Rios ( $\sim$ saleterios@ig.com.br)

Universidade de Brasília

Ana Carolina Rios Chen

Universidade de Brasília

Juliana Rios Chen

Universidade de Brasília

\section{Case Report}

Keywords: COVID-19, Lifestyle intervention, balanced nutrition, physical activity, immune system

Posted Date: June 26th, 2020

DOI: https://doi.org/10.21203/rs.3.rs-37262/v1

License: (c) (i) This work is licensed under a Creative Commons Attribution 4.0 International License.

Read Full License 


\section{Abstract}

The new disease COVID-19 is associated with high mortality, as the virus attacks our defences and our immune system. Despite the amount of research by scientists from around the world, currently, no specific antiviral treatment is recommended, and no vaccine is available. However, there is already robust research showing how to improve our immune systems through a balanced diet and lifestyle intervention in order to fight against the virus. We report a series of 10 cases of patients with COVID-19 from a poor community in Brazil who were monitored by telephone due to the lack of resources in the public health system. They received conventional medical drugs and were additionally instructed to change their eating patterns as well their lifestyles. These patients showed favourable results in their illnesses. Lifestyle interventions with balanced nutrition are not antagonistic to traditional medicine. They interact harmoniously to achieve a positive result faster. Although today we know little about the treatment of COVID-19 with drugs, we know a lot about how to improve our immune systems to help our bodies in this battle against COVID-19. Until new treatments and effective vaccines are discovered, perhaps we can revisit the teachings of the father of medicine, who 2500 years ago spoke of the importance of lifestyle interventions such as changes in nutrition, sleep and exercise for the prevention and treatment of diseases.

\section{Introduction}

Since the beginning of 2020 , the world has changed radically due to the pandemic caused by the new coronavirus, also known as COVID-19. Drastic preventive measures, including social distancing, have changed the dynamics of everyday life[1]. Strategies to deal with the infection of COVID-19 are only preventive, with the aim of reducing transmission in the community[2]. There is currently no specific antiviral treatment recommended, and no vaccine is available for this disease[2]. Although some antiviral drugs are available, the alarming increase in virus strains resistant to them highlights the need to find new antiviral compounds [3].

Almost 2,500 years ago, Hippocrates called attention to the role of nutrition, exercise and lifestyle changes in patient care[4] [5] [6]. [7]. In line with the teachings of the father of medicine, there are consistent data suggesting that the risk of being infected with viral diseases of the upper respiratory tract is high under inadequate sleep, psychosocial or physical stress, exposure to cold temperatures, inadequate nutrition and any condition that compromises the immunological system[1]. Although clinical trials are being carried out to test numerous hypotheses and different drugs in the treatment of Covid-19, an important question for the community is whether there are certain nutrients and dietary patterns that can prevent viral infection or mitigate its severity[1]. Nutrition is vital, not only for human growth and development but also for disease prevention and treatment[8]. It has been proven that balanced nutrition can help maintain immunity and is essential for the prevention and management of viral infections. Nutritional status appears to be a relevant factor that influences the outcome of COVID-19 treatment[9]. 
Studies have proven that there is potential in drugs combined with nutrition and other lifestyle interventions to increase treatment effectiveness [10].

Two other important pillars that contribute to the improvement of immunity and act against infections of the upper respiratory tract are physical exercise and adequate sleep. Sleep quality has been proven to be an important predictor of immunity and, in turn, susceptibility to the common cold. [11]. There is no scientific data on how physical activity can improve immune responses to coronaviruses, but we know that having high levels of cardiorespiratory fitness and exercising with moderate to vigorous intensity can improve immune responses to vaccination, reduce low-grade chronic inflammation and improve several immunological markers[12]. Moderate exercise induces an increase in stress hormones that act against excessive inflammation in the respiratory tract, helping to activate innate antiviral immunity by preventing an excessive immune reaction to these pathogens[13]. Skeletal muscle is an important immune regulating organ and it generates a variety of myocins, which have anti-inflammatory and immunoprotective effects. Several studies have indicated that maintaining physical activity during viral infections has immune benefits even in the elderly, reducing systemic inflammation associated with chronic age-related diseases [14].

We report the cases of 10 consecutive patients from a poor community in Brazil who were followed up by telephone and showed good results with lifestyle interventions and changes in diet associated with drug treatments. All cases were confirmed through RT-PCR exams or serological tests conducted through local health surveillance.

\section{Case Reports}

- Case 1. 53 years old, male, diabetic, overweight. He had a constant cough and fever, which improved with antipyretics. He also presented with excruciating low back pain that radiated to both lower limbs. Day 2. He got in touch with our team after he was unable to be treated at the hospital. Azithromycin $500 \mathrm{mg} / \mathrm{a}$ day for 5 days was prescribed in association with a lifestyle intervention that consisted of increased consumption of milk and yogurt, eating lots of vegetables, fruits and grains, and especially beans. To avoid fried sugary foods and red meat and to increase his hydration by consuming 2-3 litres of water/day. Light exercises were prescribed, for example: 50 minutes of daily walking, as well exposure to 20 minutes of sunlight in the morning and sleeping well. He immediately initiated these guidelines. Day 3. He referred to anosmia and ageusia, but his fever had stopped and his cough was reduced. Day 4. He experienced severe chest pain. We advised him to go to the hospital because it could be a heart attack. They performed an electrocardiogram, and the results were normal. Blood glucose was $414 \mathrm{mg} / \mathrm{dl}$. They prescribed insulin and metformin. He cough persisted, but to a lesser extent. Day 5 . He felt nausea and vomiting that could be secondary to COVID and/or side effects of the antibiotic. He increased his hydration. Day 6. He feels very well. His blood glucose was $165 \mathrm{mg} / \mathrm{dl}$. Day 7. He had a light cough and a chest X-ray was requested as obtaining a CT would be impossible. He waited 10 hours at the emergency room in the hospital close to his farm but was unable to get a chest X-ray due to the other patients who were in a worse 
condition. Normal oxygen saturation. At night his cough improved. Day 8. Complete improvement of his symptoms. Asymptomatic since then. Blood glucose 126. Day 10. Blood collection for antibody testing: COVID-19 IgG (-) IgM (+).

- Case 2. 35 years morbidly obese, male. He had a cough and fever and went to the hospital. He underwent a chest X-ray and was suspicious of COVID-19, a sample was collected for RT-PCR. They prescribed azithromycin and instructed him to return home and be isolated. Two days later, he started to experience ageusia, anosmia, asthenia and mild dyspnea. He was instructed to take 2.5 single-dose ivermectin tablets and to make a lifestyle intervention, which he started immediately. The fever subsided 24 hours later. However, he had a cough and mild respiratory distress until the 9th day of the disease; but always with normal oxygen saturation and improvements every day. Thereafter, he had complete improvement of his symptoms.

- Case 3. Health care professional, 50 years old, male. He had hypertension and was using amlodipine. Day 1. The RT-PCR test was positive. He felt anxious, with palpitations. We performed blood tests and his D-dimer was 6 times the normal value. He was prescribed 2.5 single-dose ivermectin tablets plus lifestyle intervention and saturation monitoring. He was asymptomatic 2 days later and his tests later returned to normal.

- Case 4. 27 years old, health care professional, female. No underlying pathology. Day 1. Fever, occasional cough and asthenia. On the third day she contacted us. We suggested lifestyle modification. Day 4. Her fever improved as did the coughing. She persisted with asthenia and palpitations. We asked for $\mathrm{X}$-rays and the images and her $\mathrm{O}_{2}$ saturation were normal. She was asymptomatic from the 10 th day.

- Case 5. 70 years old, female. She had a history of hypertension and heart disease. She complained of asthenia and mild dyspnoea for 3 days. Her chest $\mathrm{X}$-ray and $\mathrm{O}_{2}$ saturation were normal. She went to the hospital and was prescribed azithromycin and antipyretics. Her family contacted us and she was instructed to intervene with her lifestyle. Twenty-four hours later, her symptoms improved. On the third day, she had dizziness and vomiting and was advised that it could be due to COVID or side effects of the macrolide. She returned to the hospital but was not admitted. She had a normal chest X-ray. She took 2 ivermectin tablets. Five days later, she improved and progressed favourably and without complaints.

- Case 6. Nursing assistant, female, 41 years old. Non-specific flu-like symptoms for 4 days. Headache, asthenia, and difficulty swallowing. No fever. She took anti-inflammatory drugs on her own but did not improve. She was advised to make an intervention in her lifestyle. Day 5. She complained of anosmia and ageusia but an improved general condition. Day 6. She experienced headache and nausea. She was prescribed ivermectin plus zinc and azithromycin. Day 7. Improvement of symptoms. Day 8. No more symptoms.

- Case 7. Housewife, female, 39 years old, obese. Complaining of anosmia ageusia, runny nose and nasal burning but without fever. RT-PCR positive. On the third day she contacted us and was prescribed ivermectin plus a lifestyle intervention. Twenty-four hours later, the patient improved and was totally asymptomatic 3 days later. 
- Case 8. Overweight patient, 50 years old, experienced asthenia, intense myalgia, ageusia and anosmia. On day 2 , he was prescribed ivermectin, azithromycin and a lifestyle intervention. From then, his condition improved, and he was asymptomatic on day 6 .

- Case 9. Male, 44 years old, autoimmune disease. History of recurrent pneumonia. He had symptoms of general malaise as well as myalgia, but without fever. He did the lifestyle intervention and was asymptomatic 48 hours later. He no longer has symptoms.

- Case 10. Male, 48 years old, overweight, with an increased abdominal circumference, and major depression. Due to his disease he uses mood stabilizing drugs. Day 1 he presented with fever. He was prescribed ivermectin plus azithromycin and a lifestyle intervention. Asymptomatic by day 2 . Thereafter, he had a favourable progression.

\section{Discussion}

It is intriguing that although viral infection by COVID-19 results in a wide spectrum of symptoms, approximately $80 \%$ of patients have few or no symptoms[15] What differentiates asymptomatic patients from those who will have severe conditions? In addition to age, being male and having co-morbidities such as diabetes, obesity and hypertension, are there other factors in the lifestyle that may favour a greater severity of the disease in some more than in others? Why is it that among people in the same family, some contract the virus and others do not? What are the differences between these people's lifestyle habits? Despite the fact that the disease is still very recent, what calls attention in COVID-19 is the huge variation in the mortality rate of cases reported between countries and even between regions of the same country [15]. While these differences may, in part, be related to variations in case definitions, reporting and surveillance, they can also be attributed to underlying physiological reasons. It is likely that several factors, including age, co-morbidities, race, access to health care, genetic factors, as well as lifestyle and the complex interactions among these factors, determine the clinical course after exposure to SARS-CoV-2 [15].

Studies have shown that interventions in lifestyle can help the body fight at all stages of the diseases secondary to COVID-19[16]. Clinically, the immune responses induced by SARS-CoV-2 infection are biphasic. During the incubation stages and when the symptoms are not severe, a specific adaptive immune response is needed to eliminate the virus and prevent the disease from progressing to severe stages. Therefore, strategies to increase immune responses at this stage are very important[17]. For the development of an endogenous protective immune response in the incubation and non-severe phases, the host must be in good general health in order to elicit specific antiviral immunity[17]. The first protective phase is based on immune defence and the second harmful phase is caused by inflammation. Doctors should try to increase immune responses during the first, while suppressing it in the second phase[18], because excessive inflammation and an exaggerated immune response contribute to a cytokine storm and subsequent progression to acute lung injury/acute respiratory distress syndrome and often death[17]. 
The emerging literature on patients with COVID-19 indirectly highlights the relevance of nutrition in determining possible outcomes. However, no research to date has discussed how to adapt the currently available guidelines for nutritional therapy during illness in the specific clinical setting of COVID-19[9]. A protocol based on clinical experience and learned from the daily challenges with COVID-19 patients can help to stimulate discussions about nutrition options and lead to improvements in the standardization of nutritional approaches and in the identification of ideal care[9]. For a viral disease such as COVID-19, where there are currently no pharmacological prevention or treatment strategies and the exact timing of the end of the alarming situation is unknown, nutritional strategies to improve immunity are something to be explored[19].

Breast milk is one of the most studied foods in disease prevention and is known to protect against respiratory infections in childhood[20]. Cow's milk has also been shown to be an effective food in the prevention and treatment of viral diseases, including those caused by viruses that affect the upper airways[21]. Since 1949, Lanni has drawn attention to the beneficial effects of milk in treating flu. According to him, milk is able to inhibit haemagglutination by influenza viruses, and this inhibitory effect is a characteristic component of this food[22]. Milk is a vital nutritional source for the offspring of all mammals. In addition to its nutritional value, it is a rich source of protein and a wide variety of protective factors for the host, such as lactoferrin and lactoperoxidase that act against infectious microbes. These proteins exhibit antiviral properties and immunomodulatory effects[23] [24].

Lactoferrin is a multifunctional protein that has been extensively studied in recent decades[24]. It is believed to be the most versatile protein present in the defence of the host against injuries and infections. Due to its propensity to interact with various microbial targets, lactoferrin not only has antimicrobial properties but also modulates innate and adaptive immune responses. It can increase and decrease the activation, migration and growth of immune cells.[25]. Lactoferrin is an iron-binding glycoprotein of the transferrin family with particularly high levels in mammalian milk. It is present in large quantities in colostrum and breast milk, and in cow's milk. It is found in many biological secretions such as tears, plasma[26, 27],[28], nasal exudate, saliva, bronchial mucus and gastrointestinal fluids [29].

The consumption of lactoferrin can protect the host from viral infections by inhibiting the binding of the virus to cells, also inhibiting viral replication in cells and improving systemic immune functions[30]. The antiviral effect of lactoferrin occurs in the early stage of infection. This protein prevents the virus from entering the host cell, blocking cell receptors or binding directly to viral particles[31]. In common infections, such as cold, flu, gastroenteritis and herpes, lactoferrin mainly inhibits viral binding to target cells [30]. Lactoferrin also has the ability to modulate cytokine production from monocytes and lymphocytes during the activation by foreign or mitogenic stimuli. In addition, it can modulate chemokine recognition and lymphocyte migration potential. These factors, associated with the ability to affect the production and activity of reactive oxygen species, allow lactoferrin to serve as a unique regulator for a wide range of responses, including those involved in septic shock (for example, systemic inflammatory response syndrome) and inflammation [32]. 
In vitro studies have shown that lactoferrin significantly inhibits COVID-19[33]. In SARS-cov, lactoferrin plays an important role in preventing coronavirus invasion by increasing the activity of natural killer (NK) cells and stimulating the adhesion and aggregation of neutrophils. In the viral binding stage, lactoferrin has an important effect in preventing the preliminary interaction between host cells and SARS-cov [34]. A recent study in Rio de Janeiro found activity of lactoferrin against COVID-19. Using an antiviral assay based on the quantitative chain reaction of reverse polymerase transcription, bovine lactoferrin was found to inhibit the proliferation of COVID-19 viral progeny by up to $\sim 84.6 \%$ in African green monkey kidney epithelial cells and $\sim 68.6 \%$ in human basal alveolar epithelial cells at $1 \mathrm{mg} / \mathrm{mL}$, a concentration previously shown to have low cytotoxicity. Therefore, the data suggest that bovine lactoferrin has the potential to constitute a biochemical approach to combatting this new COVID-19 pandemic[33]. In SARScov, a severe respiratory infection, lactoferrin plays an important role in preventing the preliminary interaction between host cells and SARS-cov[34]. By investigating the role of lactoferrin in the entry of SARS pseudovirus into cells, it was proven that it inhibits pseudovirus infection and it was also able to block spike protein binding to host cells at $4{ }^{\circ} \mathrm{C}$, indicating that it exerted its inhibitory function in the viral binding stage [34].

Lactoferrin also suppresses the infiltration of inflammatory cells of the influenza virus (H1N1) into the lungs[23]. Oral administration of lactoferrin or lactoperoxidase was able to decrease the symptoms of pneumonia in mice infected with the influenza virus, suppressing the infiltration of inflammatory cells in the lung[23]. The rats that received lactoferrin showed less worsening in lung injury on the sixth day after infection compared to the rats that received only water. In addition, the number of infiltrated leukocytes recovered from bronchoalveolar lavage fluid was also much lower in mice that received lactoferrin or lactoperoxidase[23]. It interacts with the influenza $A$ virus haemagglutinin and prevents infection by different viral subtypes of $\mathrm{H} 1$ and $\mathrm{H} 3$ [35]. Lactoferrin suppresses cytopathic effects by inhibiting haemagglutination and virus-induced infection[3,36]. The interaction of lactoferrin with influenza A H1N1 haemagglutinin at low $\mathrm{pH}$ induces changes that stabilize the conformation of haemagglutinin, resulting in the inhibition of viral activity [35]. In addition, bovine lactoferrin prevents apoptosis (or death) induced by the influenza virus as well as the cytopathic effects of the virus when incubated with cells after the adsorption step [3]. The processing of lactoperoxidase, lysozyme, lactoferrin and immunoglobulin activate several bioactive peptides that can potentiate physiological effects on the human body, especially on the immune system, through their antimicrobial and antiviral activities [37].

It has been proven that other viruses are also inhibited by the action of the lactoferrin. This protein and a small peptide fragment from its N-terminal domain effectively block the entry of the herpes virus into the host cell and appear to have activities that stimulate the immune system [38]. One study proved that consumption of lactoferrin also reduced the incidence of noroviral gastroenteritis in children[30]. It is a potent inhibitor of several enveloped and naked viruses, such as rotavirus, enterovirus and adenovirus. Because it is resistant to digestion, lactoferrin has a dual role, preventing adenovirus from binding to intestinal cells by binding to viral particles and inhibiting a post-adsorption step[39]. In poliovirus, the action occurs due to interference in an initial stage of infection, but when the bovine lactoferrin molecule 
is saturated with $\mathrm{Zn}^{+2}$ ions, it is also capable of inhibiting viral replication after the viral adsorption phase[39]. The anti-adenovirus action of lactoferrin occurs during binding of the virus to cell membranes through competition for common glycosaminoglycan receptors and through a specific interaction with viral structural polypeptides[39]. Lactoferrin is an excellent natural candidate against enteric viral diseases, as it acts mainly by preventing the adsorption and internalization of the virus in cells through specific binding to cellular receptors and/or viral particles. [39]

HIV-1 replication and syncytium formation were efficiently inhibited in a dose-dependent manner by lactoferrins [28]. Bovine lactoferrin markedly inhibited HIV-1 replication when added before HIV infection or during the virus adsorption stage, thus suggesting a mechanism of action for HIV binding or entry into cells. The potent antiviral effect and the high selectivity index exhibited by bovine lactoferrin suggest that this protein has an important role in inhibiting the early interaction of HIV with cells[28].

Lactoferrin also significantly inhibited infection by the four dengue serotypes. It blocked the link between the dengue virus and the cell membrane, interacting with heparan sulfate, the specific dendritic intercellular adhesion molecule of non-integrin cell 3 and low density lipoprotein receptors. In addition to bovine lactoferrin inhibiting dengue virus infection, it also decreased morbidity [40]. Bovine lactoferrin exerted a strong dose-dependent inhibitory effect on the infection of cells by the arboviruses of Zika and Chikungunha, reducing the efficiency of infection by up to $80 \%$, without expression and without cytotoxicity. This antiviral activity occurs at the level of the entry and exit of the viral particles[41]. The antiviral effects of lactoferrin have also been observed against other viruses such as the human papilloma virus HPV, which causes cervical cancer[42], the hepatitis C virus, herpes and rotavirus[42]. Lactoferrin also suppresses poliovirus infection of renal cells in monkey embryos, attaching to susceptible cell surfaces and preventing viral adhesion[43]. Lactoferrin has a broad spectrum of action and exhibits antiviral activity against DNA and RNA viruses, including rotavirus, respiratory syncytial virus, herpes virus and HIV [31] and increases the potential for inhibiting HIV reverse transcriptase [44].

Cow's milk and yogurt are great dietary sources of lactoferrin. The milk of other mammals also contains lactoferrin. The concentrations of lactoferrin obtained in different animal milks (cow, buffalo and goat), as well as in milk and yogurt, are much higher in raw buffalo milk and raw cow's milk than in fresh commercial samples of cow's and goat's milk. The lactoferrin found in raw cow's milk is approximately $10 \times$ the amount found in UHT milk purchased on the market (raw milk has $182.4 \mathrm{mg} / \mathrm{L}$ while UHT milk has $18.3 \mathrm{mg} / \mathrm{L}$ and yogurt has $8 \mathrm{mg} / \mathrm{L}$ ) [45]. Bovine lactoferrin is homologous to human lactoferrin[16]. In addition to lactoferrin, studies have shown that vitamin $D$ can have an antiviral action against COVID-19, as well as against the influenza virus [46], and milk is one of the main foods rich in vitamin D[47].

One condiment that can be added to the diet in this pandemic is turmeric. Saffron can be used to treat patients with COVID-19 and this may be behind the differences in high mortality among western countries that do not consume it when compared to countries that consume it in large quantities [48]. The antiviral properties of curcumin have already been extensively studied. Turmeric has been used for centuries in traditional Asian medicine and has been proven to have anti-inflammatory, antiangiogenic and 
antineoplastic actions. It also has antiviral and antibacterial activity against various human pathogens, such as influenza, hepatitis C, HIV and strains of Staphylococcus, Streptococcus and Pseudomonas[49]. Curcumin can directly inactivate the influenza A virus, block its adsorption and inhibit its proliferation and replication[50]. In one study, curcumin significantly increased the survival rate of mice, reduced the production of inflammatory cytokines and histopathological changes in the lungs after influenza $A$ infection[50].

Another important food is beans. Beans act against malaria pathogens in a similar way to chloroquine. Haemoglobin $E$ in the presence of oxidative substances from fava beans can protect individuals against Plasmodium falciparum which causes malaria. In Thailand a study found an association between haemoglobin E and Plasmodium falciparum malaria and the action of beans; The study suggested a possible synergistic protective effect of haemoglobin $\mathrm{E}$ on the risk of $P$. falciparum malaria in individuals who consumed fava beans[51]. In other words, it showed a similar action to quinine-based antimalarial drugs, some of which were used in people infected with COVID-19, such as hydroxychloroquine.

The disease caused by COVID-19 can predispose patients to thrombotic diseases, in venous and arterial circulation, due to excessive inflammation, platelet activation and endothelial dysfunction [52]. One study tested several types of fruits, vegetables and regular exercise for their effects on global thrombotic status. There was evidence of benefit of an anti-thrombotic diet rich in fruits and vegetables and regular exercise in reducing thrombotic status. This could be a simple and economical way to prevent arterial thrombotic diseases[53]. Fruits, vegetables and legumes contain high levels of phytochemicals that show antiinflammatory effects. The phenolics and triterpenoids in fruits and vegetables and the lectins and peptides in legumes are responsible for the anti-inflammatory activity in these foods[54]. In fact, epidemiological evidence suggests that a diet with large amounts of fruits and vegetables and little meat consumption can substantially reduce the risk of venous thromboembolism; vegetarian, vegan or Mediterranean diets favourably affect serum markers of haemostasis and inflammation [55]. The levels of homocysteine, factor VIII and von Willebrand factor, risk factors for venous thromboembolism, are also influenced by food intake. Meat intake is positively associated with the incidence of thromboembolism. It has been observed that a diet that includes more plant and fish foods and less red and processed meat is associated with less risk of thromboembolism. The prevention of non-communicable diseases related to nutrition has recently gained prominence [56].

Good hydration is associated with a reduction in infections, hypertension, fatal coronary heart disease and venous thromboembolism[56]. It has been proven that dehydration predisposes to venous thromboembolism. One study evaluated the relationship between biochemical indices of dehydration and venous thromboembolism after an acute ischaemic stroke. Dehydration criteria correlated with the risk of thromboembolism[57]. Hydration is also essential in the fight against the coronavirus. The coronavirus was found to be quite stable at $\mathrm{pH} 6.0$ and $37^{\circ} \mathrm{C}$ (half-life, approximately 24 hours) but was rapidly and irreversibly inactivated by a pH increase to 8.0 and $37^{\circ} \mathrm{C}$ (half-life). approximately 30 minutes) [58]. It is good to remember that diet and hydration play a role in adjusting the $\mathrm{pH}$ of the blood[59]. 
Inflammation is at the heart of the complications caused by this virus. In addition, it is good to remember that peripheral inflammation caused by COVID-19 can have long-term consequences that can lead to neurodegenerative dementia and disease through neuroinflammatory mechanisms that can be potentiated and aggravated by an unhealthy diet, especially in the most vulnerable people[60]. In addition to investing in healthy foods, we have to restrict inflammatory foods. The consumption of a western type diet activates the innate immune system and impairs adaptive immunity, leading to chronic inflammation, reducing the host's defences against COVID-19[60]. It is also important to remember that the high rate of consumption of diets rich in saturated fats such as red meat, sugars and refined carbohydrates worldwide, contributes to the prevalence of obesity and type 2 diabetes and can put these populations at increased risk for developing severe COVID-19 and lead to higher mortality[60]. At the moment when we are going through this pandemic, it is essential to consider the impact of lifestyle habits, such as the consumption of unhealthy diets, on susceptibility to COVID-19 as well as on its recovery. Therefore, individuals should refrain from eating foods rich in saturated fats and sugar and instead consume large amounts of fibre, whole grains, unsaturated fats and antioxidants to increase immune function[60, 61].

To strengthen the immune system the practice of physical activity is very important. Moderate-intensity exercise reduces inflammation, improves immune function and decreases the risk and severity of respiratory infection [13]. It acts by elevating stress hormones that negatively regulates excessive inflammation in the respiratory tract and helps in the activation of innate antiviral immunity, shifting the immune response to a Th2 profile, thus balancing the Th1/Th2 responses to prevent an excessive Th1 immune reaction to these pathogens[62]. It also increases immune defences through frequent mobilization and redistribution of effector lymphocytes. Billions of lymphocytes are mobilized in response to just one exercise[14]. This mobilization is mediated by catecholamine. Lymphocytes capable of migrating into the tissues are prepared to recognize and kill virus-infected cells. The anti-virus specific memory $T$ cells mobilized through exercise exhibit enhanced proliferation responses to viral antigens, such as those derived from cytomegalovirus, Epstein Barr and herpes simplex V-1, and non-latent viruses, such as adenovirus [14]. Based on the available evidence, moderate-intensity physical training should be used as a complement to other preventive measures against viral respiratory tract infection. [62]. It can provide an adequate response to stress that leads to immunopotentiation and anti-inflammatory actions, resulting in improved recovery and survival after respiratory viral infection. [62].

Another lifestyle intervention that helps with immune defences is vitamin $D$ obtained through the sunlight. Solar radiation triggers a robust production of vitamin $D$ in the skin; Vitamin $D$ deficiency is common in winter, which explains the seasonality of the flu epidemic[63]. Activated vitamin D $1.25(\mathrm{OH})$ 2D, a steroid hormone, has profound effects on human immunity, modulating the immune system, preventing the excessive expression of inflammatory cytokines and increasing the potential for 'oxidative explosion' of macrophages[63]. Vitamin D also stimulates the expression of potent antimicrobial peptides, found in neutrophils, monocytes, natural killer cells and in epithelial cells that line the respiratory tract, where they play an important role in protecting the lung against infections[63]. There is a hypothesis that there is a potential association between average vitamin $D$ levels in several countries, a high number 
of cases and mortality caused by COVID-19. Average vitamin D levels in 20 European countries and morbidity and mortality caused by COVID-19 were evaluated. It was observed that vitamin D levels are severely low in the ageing population, especially in Spain, Italy and Switzerland, and this is also the most vulnerable group of the population in relation to COVID-19 [64]. Few foods naturally contain vitamin D; dermal synthesis is the main source. Vitamin D3 is synthesized non-enzymatically in the skin during exposure to ultraviolet B radiation (UVB) in sunlight. Vitamin D3 is inactive and requires enzymatic conversion in the liver and kidneys to form the active form, 1,25-dihydroxyvitamin D. Ageing decreases the skin's ability to produce vitamin D[65].

Sleep and the circadian rhythm also have a strong regulatory influence on immune functions. Investigations of the sleep-wake cycle showed that immunological parameters such as the number of $T$ cells and the production of pro-inflammatory cytokines exhibit peaks during early night sleep [66]. The number of circulating immune cells with immediate effector functions, as well as the number of natural cytotoxic killer cells, and the peak of cytokine anti-inflammatory activity occurs during daytime waking. Sleep facilitates the extravasation of $T$ cells and their redistribution between lymph nodes. In addition, studies have shown an influence of sleep on cytokines, promoting the interaction between antigenpresenting cells and helper T cells, such as interleukin-12[66].

Regarding azithromycin and ivermectin, we used ivermectin in the viral proliferation phase because it showed effects in vitro against COVID-19[67], and it is quite cheap and affordable. Azithromycin has been prescribed because studies show that it helps modulate the immune system response as well virus elimination [68].

\section{Conclusion}

The COVID-19 pandemic's current situation requires that we search for any means to contain and control it. Thus, lifestyle interventions, such as a healthier diet, exercise and sleep, can present themselves as a tool to help overcome this disease associated with the available drugs. Despite the intensive work of thousands of excellent and brilliant scientists researching COVID around the world, we are not getting the answers we need to deal with COVID-19. Robust research has proven the role of food and lifestyle intervention in the treatment and prevention of diseases, including viral infections of the upper airways. In the exceptional situation we find ourselves in, where there are difficulties in finding a treatment quickly, and the number of deaths continues to increase due to the complications caused by COVID-19, it might be time to turn to this evidence-based scientific knowledge to improve our immune system in the fight against COVID-19. Although this is not the definitive solution to the problem, it will certainly be another soldier in the fight against this virus.

\section{Declarations}

\section{Conflicting of interests}


The authors declare that they have no competing interests

\section{Funding}

The author(s) received no financial support for the research, authorship and/or publication of this article.

\section{Informed consent}

Written informed consent was obtained from the patient(s) for their anonymized information to be published in this article.

\section{Acknowledgment}

To all the patients

\section{References}

1. K. Kalantar-Zadeh, L. W. Moore, "Impact of Nutrition and Diet on COVID-19 Infection and Implications for Kidney Health and Kidney Disease Management", J Ren Nutr, vol. 30, no. 3, pp. 179-81, 2020.

2. M. Cascella, M. Rajnik, A. Cuomo, et al. Features, Evaluation and Treatment Coronavirus (COVID-19). StatPearls. Treasure Island (FL)2020.

3. A. Pietrantoni, M. G. Ammendolia, F. Superti, "Bovine lactoferrin: involvement of metal saturation and carbohydrates in the inhibition of influenza virus infection", Biochemistry and cell biology = Biochimie et biologie cellulaire, vol. 90, no. 3, pp. 442-8, 2012.

4. C. Yapijakis, "Hippocrates of Kos, the father of clinical medicine, and Asclepiades of Bithynia, the father of molecular medicine. Review", In Vivo, vol. 23, no. 4, pp. 507-14, 2009.

5. M. Lucock, "Is folic acid the ultimate functional food component for disease prevention?", BMJ (Clinical research ed), vol. 328, no. 7433, pp. 211-4, 2004.

6. C. F. Kleisiaris, C. Sfakianakis, I. V. Papathanasiou, "Health care practices in ancient Greece: The Hippocratic ideal", Journal of medical ethics and history of medicine, vol. 7, no., pp. 6-, 2014.

7. E. Tsiompanou, S. G. Marketos, "Hippocrates: timeless still", J R Soc Med, vol. 106, no. 7, pp. 288-92, 2013.

8. S. D. Ohlhorst, R. Russell, D. Bier, et al., "Nutrition research to affect food and a healthy life span", $J$ Nutr, vol. 143, no. 8, pp. 1349-54, 2013.

9. A. Laviano, A. Koverech, M. Zanetti, "Nutrition support in the time of SARS-CoV-2 (COVID-19)", Nutrition, vol., no., pp. 110834, 2020.

10. R. F. Witkamp, K. van Norren, "Let thy food be thy medicine.... when possible", European journal of pharmacology, vol. 836, no., pp. 102-14, 2018.

11. S. Cohen, W. J. Doyle, C. M. Alper, et al., "Sleep habits and susceptibility to the common cold", Archives of internal medicine, vol. 169, no. 1, pp. 62-7, 2009. 
12. R. J. Simpson, E. Katsanis, "The immunological case for staying active during the COVID-19 pandemic", Brain, behavior, and immunity, vol., no., pp. S0889-1591(20)30573-0, 2020.

13. S. A. Martin, B. D. Pence, J. A. Woods, "Exercise and respiratory tract viral infections", Exercise and sport sciences reviews, vol. 37, no. 4, pp. 157-64, 2009.

14. N. A. Duggal, G. Niemiro, S. D. R. Harridge, et al., "Can physical activity ameliorate immunosenescence and thereby reduce age-related multi-morbidity?", Nature reviews Immunology, vol. 19, no. 9, pp. 563-72, 2019.

15. P. E. Marik, P. Kory, J. Varon, "Does vitamin D status impact mortality from SARS-CoV-2 infection?", Med Drug Discov, vol., no., pp. 100041-, 2020.

16. Y. W. Park, M. S. Nam, "Bioactive Peptides in Milk and Dairy Products: A Review", Korean J Food Sci Anim Resour, vol. 35, no. 6, pp. 831-40, 2015.

17. R. Zhang, X. Wang, L. Ni, et al., "COVID-19: Melatonin as a potential adjuvant treatment", Life sciences, vol. 250, no., pp. 117583-, 2020.

18. Y. Shi, Y. Wang, C. Shao, et al., "COVID-19 infection: the perspectives on immune responses", Cell Death Differ, vol., no., pp. 1-4, 2020.

19. R. Jayawardena, P. Sooriyaarachchi, M. Chourdakis, et al., "Enhancing immunity in viral infections, with special emphasis on COVID-19: A review", Diabetes Metab Syndr, vol. 14, no. 4, pp. 367-82, 2020.

20. J. T. May, "Microbial contaminants and antimicrobial properties of human milk", Microbiological sciences, vol. 5, no. 2, pp. 42-6, 1988.

21. E. Prahoveanu, V. Esanu, N. Cajal, et al., "Effect of several unpurified milk preparations on experimental influenza infection in mice", Virologie, vol. 37, no. 1, pp. 49-53, 1986.

22. F. Lanni, Y. T. Lanni, J. W. Beard, "Inhibitory effect of cow's milk on influenza virus hemagglutination", Proc Soc Exp Biol Med, vol. 72, no. 1, pp. 227-32, 1949.

23. K. Shin, H. Wakabayashi, K. Yamauchi, et al., "Effects of orally administered bovine lactoferrin and lactoperoxidase on influenza virus infection in mice", J Med Microbiol, vol. 54, no. Pt 8, pp. 717-23, 2005.

24. H. Jenssen, R. E. Hancock, "Antimicrobial properties of lactoferrin", Biochimie, vol. 91, no. 1, pp. 19-29, 2009.

25. D. Legrand, "Overview of Lactoferrin as a Natural Immune Modulator", J Pediatr, vol. 173 Suppl, no., pp. S10-5, 2016.

26. F. Giansanti, G. Panella, L. Leboffe, et al., "Lactoferrin from Milk: Nutraceutical and Pharmacological Properties", Pharmaceuticals (Basel), vol. 9, no. 4, pp. 61, 2016.

27. A. Pierce, D. Legrand, J. Mazurier, "[Lactoferrin: a multifunctional protein]", Medecine sciences : $M / S$, vol. 25, no. 4, pp. 361-9, 2009.

28. P. Puddu, P. Borghi, S. Gessani, et al., "Antiviral effect of bovine lactoferrin saturated with metal ions on early steps of human immunodeficiency virus type 1 infection", Int J Biochem Cell Biol, vol. 30, no. 9, pp. 1055-62, 1998. 
29. E. D. Weinberg, "The therapeutic potential of lactoferrin", Expert opinion on investigational drugs, vol. 12, no. 5, pp. 841-51, 2003.

30. H. Wakabayashi, H. Oda, K. Yamauchi, et al., "Lactoferrin for prevention of common viral infections", Journal of infection and chemotherapy : official journal of the Japan Society of Chemotherapy, vol. 20, no. 11, pp. 666-71, 2014.

31. B. W. van der Strate, L. Beljaars, G. Molema, et al., "Antiviral activities of lactoferrin", Antiviral research, vol. 52, no. 3, pp. 225-39, 2001.

32. J. K. Actor, S.-A. Hwang, M. L. Kruzel, "Lactoferrin as a natural immune modulator", Curr Pharm Des, vol. 15, no. 17, pp. 1956-73, 2009.

33. C. A. M. d. C. A. d. R. M. B. C. C. I. P. d. S. J. S. P. d. C. C. B. R. G. C. A. B. Matheus Augusto Patricio de Almeida; Vanessa Pimenta Rocha; Andréa Marques Vieira da Silva; Juliana Gil Melgaço; Patrícia Cristina da Costa Neves; Tamiris Azamor da Costa Barros; Ana Paula Dinis Ano Bom; Marilda Mendonça Siqueira Sotiris Missailidis; Rafael Braga Gonçalves. In Vitro Inhibition of SARS-CoV-2 Infection by Bovine Lactoferrin 2020 [Available from: https://www.biorxiv.org/content/10.1101/2020.05.13.093781v1.

34. J. Lang, N. Yang, J. Deng, et al., "Inhibition of SARS pseudovirus cell entry by lactoferrin binding to heparan sulfate proteoglycans", PloS one, vol. 6, no. 8, pp. e23710-e, 2011.

35. F. Superti, M. Agamennone, A. Pietrantoni, et al., "Bovine Lactoferrin Prevents Influenza A Virus Infection by Interfering with the Fusogenic Function of Viral Hemagglutinin", Viruses, vol. 11, no. 1, pp. 51, 2019.

36. M. G. Ammendolia, M. Agamennone, A. Pietrantoni, et al., "Bovine lactoferrin-derived peptides as novel broad-spectrum inhibitors of influenza virus", Pathog Glob Health, vol. 106, no. 1, pp. 12-9, 2012.

37. A. R. Madureira, T. Tavares, A. M. Gomes, et al., "Invited review: physiological properties of bioactive peptides obtained from whey proteins", Journal of dairy science, vol. 93, no. 2, pp. 437-55, 2010.

38. H. Jenssen, "Anti herpes simplex virus activity of lactoferrin/lactoferricin -- an example of antiviral activity of antimicrobial protein/peptide", Cellular and molecular life sciences : CMLS, vol. 62, no. 24, pp. 3002-13, 2005.

39. L. Seganti, A. M. Di Biase, M. Marchetti, et al., "Antiviral activity of lactoferrin towards naked viruses", Biometals : an international journal on the role of metal ions in biology, biochemistry, and medicine, vol. 17, no. 3, pp. 295-9, 2004.

40. J.-M. Chen, Y.-C. Fan, J.-W. Lin, et al., "Bovine Lactoferrin Inhibits Dengue Virus Infectivity by Interacting with Heparan Sulfate, Low-Density Lipoprotein Receptor, and DC-SIGN", Int J Mol Sci, vol. 18, no. 9, pp. 1957, 2017.

41. C. A. M. Carvalho, S. M. M. Casseb, R. B. Gonçalves, et al., "Bovine lactoferrin activity against Chikungunya and Zika viruses", The Journal of general virology, vol. 98, no. 7, pp. 1749-54, 2017.

42. N. Mistry, P. Drobni, J. Naslund, et al., "The anti-papillomavirus activity of human and bovine lactoferricin", Antiviral research, vol. 75, no. 3, pp. 258-65, 2007. 
43. K. B. McCann, A. Lee, J. Wan, et al., "The effect of bovine lactoferrin and lactoferricin B on the ability of feline calicivirus (a norovirus surrogate) and poliovirus to infect cell cultures", Journal of applied microbiology, vol. 95, no. 5, pp. 1026-33, 2003.

44. H. Wang, X. Ye, T. B. Ng, "First demonstration of an inhibitory activity of milk proteins against human immunodeficiency virus-1 reverse transcriptase and the effect of succinylation", Life sciences, vol. 67, no. 22, pp. 2745-52, 2000.

45. L. Campanella, E. Martini, M. Pintore, et al., "Determination of lactoferrin and immunoglobulin g in animal milks by new immunosensors", Sensors (Basel), vol. 9, no. 3, pp. 2202-21, 2009.

46. W. B. Grant, H. Lahore, S. L. McDonnell, et al., "Evidence that Vitamin D Supplementation Could Reduce Risk of Influenza and COVID-19 Infections and Deaths", Nutrients, vol. 12, no. 4, pp., 2020.

47. A. Schmid, B. Walther, "Natural vitamin D content in animal products", Adv Nutr, vol. 4, no. 4, pp. 45362, 2013.

48. F. A. C. Rocha, M. R. de Assis, "Curcumin as a potential treatment for COVID-19", Phytotherapy Research, vol. n/a, no. n/a, pp., 2020.

49. D. Praditya, L. Kirchhoff, J. Brüning, et al., "Anti-infective Properties of the Golden Spice Curcumin", Front Microbiol, vol. 10, no., pp. 912-, 2019.

50. J. Dai, L. Gu, Y. Su, et al., "Inhibition of curcumin on influenza A virus infection and influenzal pneumonia via oxidative stress, TLR2/4, p38/JNK MAPK and NF-kappaB pathways", International immunopharmacology, vol. 54, no., pp. 177-87, 2018.

51. D. Kitayaporn, K. E. Nelson, P. Charoenlarp, et al., "Haemoglobin-E in the presence of oxidative substances from fava bean may be protective against Plasmodium falciparum malaria", Transactions of the Royal Society of Tropical Medicine and Hygiene, vol. 86, no. 3, pp. 240-4, 1992.

52. B. Bikdeli, M. V. Madhavan, D. Jimenez, et al., "COVID-19 and Thrombotic or Thromboembolic Disease: Implications for Prevention, Antithrombotic Therapy, and Follow-up", Journal of the American College of Cardiology, vol., no., pp., 2020.

53. J. Yamamoto, Y. ljiri, H. Ikarugi, et al., "Prevention of thrombotic disorders by antithrombotic diet and exercise: evidence by using global thrombosis tests", Future Sci OA, vol. 4, no. 4, pp. FSO285-FSO, 2018.

54. F. Zhu, B. Du, B. Xu, "Anti-inflammatory effects of phytochemicals from fruits, vegetables, and food legumes: A review", Crit Rev Food Sci Nutr, vol. 58, no. 8, pp. 1260-70, 2018.

55. D. K. Cundiff, P. S. Agutter, P. C. Malone, et al., "Diet as prophylaxis and treatment for venous thromboembolism?", Theor Biol Med Model, vol. 7, no., pp. 31-, 2010.

56. B. M. Popkin, K. E. D'Anci, I. H. Rosenberg, "Water, hydration, and health", Nutrition reviews, vol. 68, no. 8, pp. 439-58, 2010.

57. J. Kelly, B. J. Hunt, R. R. Lewis, et al., "Dehydration and venous thromboembolism after acute stroke", QJM : monthly journal of the Association of Physicians, vol. 97, no. 5, pp. 293-6, 2004. 
58. L. S. Sturman, C. S. Ricard, K. V. Holmes, "Conformational change of the coronavirus peplomer glycoprotein at $\mathrm{pH} 8.0$ and 37 degrees $\mathrm{C}$ correlates with virus aggregation and virus-induced cell fusion", Journal of virology, vol. 64, no. 6, pp. 3042-50, 1990.

59. H. A.-L. Mousa, "Prevention and Treatment of Influenza, Influenza-Like Illness, and Common Cold by Herbal, Complementary, and Natural Therapies", J Evid Based Complementary Altern Med, vol. 22, no. 1, pp. 166-74, 2017.

60. M. J. Butler, R. M. Barrientos, "The impact of nutrition on COVID-19 susceptibility and long-term consequences", Brain, behavior, and immunity, vol., no., pp., 2020.

61. R. M. Connaughton, A. M. McMorrow, F. C. McGillicuddy, et al., "Impact of anti-inflammatory nutrients on obesity-associated metabolic-inflammation from childhood through to adulthood", Proc Nutr Soc, vol. 75, no. 2, pp. 115-24, 2016.

62. S. A. Martin, B. D. Pence, J. A. Woods, "Exercise and respiratory tract viral infections", Exerc Sport Sci Rev, vol. 37, no. 4, pp. 157-64, 2009.

63. J. J. Cannell, R. Vieth, J. C. Umhau, et al., "Epidemic influenza and vitamin D", Epidemiology and infection, vol. 134, no. 6, pp. 1129-40, 2006.

64. P. C. Ilie, S. Stefanescu, L. Smith, "The role of vitamin D in the prevention of coronavirus disease 2019 infection and mortality", Aging clinical and experimental research, vol., no., pp. 1-4, 2020.

65. J. MacLaughlin, M. F. Holick, "Aging decreases the capacity of human skin to produce vitamin D3", The Journal of clinical investigation, vol. 76, no. 4, pp. 1536-8, 1985.

66. L. Besedovsky, T. Lange, J. Born, "Sleep and immune function", Pflugers Arch, vol. 463, no. 1, pp. 12137, 2012.

67. L. Caly, J. D. Druce, M. G. Catton, et al., "The FDA-approved drug ivermectin inhibits the replication of SARS-CoV-2 in vitro", Antiviral research, vol. 178, no., pp. 104787, 2020.

68. P. Gautret, J. C. Lagier, P. Parola, et al., "Hydroxychloroquine and azithromycin as a treatment of COVID-19: results of an open-label non-randomized clinical trial", International journal of antimicrobial agents, vol., no., pp. 105949, 2020. 